\title{
Ligadura de arteria carótida común y exsanguinación como modelo experimental de isquemia cerebral focal en ratas
}

\section{Ligation of common carotid artery and exsanguination as experimental model of focal cerebral ischemia in rats}

\author{
Luz Marina Guerrero Espino \\ Universidad Nacional de Trujillo, Calle Salaverry Nº545-Trujillo, Perú
}

DOI: https://doi.org/10.33017/RevECIPeru2013.0017/

\section{Resumen}

La isquemia cerebral es el proceso fisiopatológico caracterizado por la disfunción de una porción del tejido cerebral secundario a la disminución del flujo en una arteria cerebral concreta. La mejor herramienta que se dispone hoy para el estudio de la fisiopatología de la isquemia cerebral son los modelos experimentales, que permiten abordar de manera simple el cuidado de esta afección que se caracteriza por su complejidad y heterogeneidad. Considerando que las investigaciones sobre este campo es ilimitada, no solo por la trascendencia sino por el costo social de la enfermedad ,el estudio de nuevas metodologías experimentales permitirán alcanzar mejores herramientas con rigor científico para el conocimiento fisiopatológico, terapéutico y preventivo de la enfermedad isquémica cerebral.Por lo que nos propusimos determinar si la ligadura de arteria carótida común izquierda y exsanguinación puede ser utilizada como modelo de isquemia cerebral focal en ratas. Se usaron 36 ratas, machos de 8 a 9 semanas de edad, con $200 \pm 20 \mathrm{gr}$ de peso, que se mantuvieron en condiciones estándar de iluminación, ciclos diarios de 12 horas de luz y 12 de oscuridad, a una temperatura ambiente de $24-25^{\circ} \mathrm{C}$, los especímenes fueron distribuidas en dos grupos: Grupo Sham o seudo operado y Grupo Experimental (operado). Se procedió a la sangría por punción cardiaca, y se extrajo de cada rata el $10 \%$ del volumen de sangre circulante. Bajo condiciones de asepsia y antisepsia, se realizó una incisión en la línea media del cuello y diseccionó la piel, tejido subcutáneo y músculo hasta lograr identificar la arteria carótida común (ACC) izquierda y se ligó con sutura Nylon 5-0 para interrumpir la circulación sanguínea de manera ipsilateral, se suturó la herida y se administró lidocaína, finalmente se dejó descansar al animal a una temperatura ligeramente mayor a la ambiental en cámaras previamente confeccionadas. Luego de 24 horas de aplicar la cirugía, se sacrificaron los especímenes por sobredosis de anestésico y se realizó la remoción del cerebro y el análisis histopatológico de secciones coronales en tres regiones neocortex, hipocampo y ganglios basales, por ser las áreas más vulnerables a daño en una lesión isquémica. Se encontró que las mayores características neuronales que indican daño celular se manifestaron en el Grupo Experimental con un p<0.01 y la zona con mayor incidencia de daño es el ganglio basal, $p<0.01$. Se concluyó que la ligadura de arteria carótida común izquierda y ex sanguinación produce daños neuronales localizados y la zona más afectada es el ganglio basal.

Descriptores: isquemia cerebral focal, ligadura arteria carótida común ex sanguinación.

\section{Abstract}

Cerebral ischemia is the pathophysiological process characterized by dysfunction of a portion of brain tissue secondary to decreased flow in specific brain artery. The best tool available today to study the pathophysiology of cerebral ischemia are experimental models, which allow a simple way to address the care of the condition which is characterized by complexity and heterogeneity. Whereas research on this field is unlimited, not only because of the importance but by the social cost of the disease, the study of new experimental methodologies 
to achieve better tools for scientific rigor in pathophysiological knowledge, treatment and prevention of cerebral ischemic disease. So we set out to determine whether the common carotid artery ligation and ex sanguination left can be used as a model of focal cerebral ischemia in rats. We used 36 male rats of 8 to 9 weeks of age, with $200 \pm 20 \mathrm{~g}$ of weight, were maintained in standard lighting conditions, daily cycles of 12 hours of light and 12 of darkness were used at an ambient temperature of $24-25^{\circ} \mathrm{C}$, the specimens were divided into two groups: Sham Groups and Experimental Group (operated). We proceeded to the bleeding via cardiac puncture, and extracted from each rat $10 \%$ of circulating blood volume. Under aseptic and antiseptic conditions, an incision was made in the midline of the neck and dissected the skin, subcutaneous tissue and muscle to be able to identify the left common carotid artery (CCA) and ligated with 5-0 nylon suture to interrupt circulation way of ipsilateral blood, the wound was sutured and received lidocaine finally allowed to rest the animal at a slightly higher environmental chambers previously made temperature. After 24 hours of surgery applied, the specimens were sacrificed by overdose of anesthetic and removal of the brain and histopathological analysis of coronal sections in three regions neocortex, hippocampus and basal ganglia was performed, being the most vulnerable areas to damage ischemic injury. It was found that the major characteristics that indicate neuronal cell damage demonstrated in the experimental group with $p<0.01$ and the area with the highest incidence of injury is the basal ganglia, $p<0.01$. It was concluded that ligation of left common carotid artery and ex sanguinación produces localized neuronal damage and the most affected area is the basal ganglia.

\section{Keywords: Focal cerebral ischemia, ligation of left common carotid artery and ex sanguination}

\section{Introducción}

La isquemia cerebral es el proceso fisiopatológico caracterizado por la disfunción de una porción del tejido cerebral secundario a la disminución del flujo en una arteria cerebral concreta. Se manifiesta por la aparición súbita de déficits neurológicos transitorios o permanentes. La evolución de la isquemia que persiste por debajo de un umbral de flujo conlleva la necrosis del tejido y el desarrollo del infarto cerebral $[1,2,3]$.

En la población adulta mayor, la principal causa de los infartos isquémicos es la oclusión aterotrombótica o cardioembólica de una arteria cerebral, mientras que en la población más joven es el vasoespasmo secundaria a una hemorragia subaracnoidea, existe otro grupo vulnerable, el neonato, por presentar hipoxia isquémica al nacer, denominada encefalopatía neonatal [4].

En otro grupo de pacientes aparecen síntomas similares pero cuya causa es la rotura intracraneal de una arteria, con la consecuente hemorragia intra parenquimatosa; estas lesiones se denominan "infartos hemorrágicos". Todo este conjunto de enfermedades se agrupa bajo el término "patología vascular cerebral" $[1,5]$.

Estudios en España, refieren que el $85 \%$ de los infartos observados en la práctica clínica son isquémicos y sólo un 15\% son de tipo hemorrágicos, según datos del Instituto de Estadística, las enfermedades vasculares cerebrales generan en ese país entre 120,000 y 130,000 nuevos casos al año y más de la mitad de los pacientes quedan discapacitados o fallecen. En los países denominados desarrollados son la primera causa de incapacidad permanente en adultos y la tercera causa de muerte, aproximadamente el $30 \%$ de los pacientes que ha sufrido un infarto cerebral queda con incapacidades graves o permanentes y la mayor parte del resto de los supervivientes presenta

dificultades funcionales. Se trata de una patología con una gran repercusión social ya que supone una carga económica muy elevada, además conforme la población envejece, su prevalencia aumenta. En los países occidentales, después de las enfermedades cardiovasculares y las neoplasias, la patología cerebrovascular constituye la tercera y para unos países la cuarta e incluso la quinta causa de mortalidad. La enfermedad cerebrovascular está entre las tres principales causas de muerte, constituye el $10 \%$ de todas ellas [6].

Actualmente en los Estados Unidos de Norte América se obtiene la cifra de un costo promedio por paciente de $\$ 46000$ en un hospital importante de Chicago, con un gasto anual de 42 billones de dólares. Este problema se acentúa más aún por lo que el paciente dejará de producir como trabajador. El costo de capital humano en el año 1993 fue calculado en 25000 millones de dólares al año [7]

En el Perú no tenemos cifras al respecto, pero, no obstante, debemos señalar que el costo de capital humano debe ser alto, sabiendo que la edad promedio de la aparición de enfermedad cerebrovascular es entre los 40 y 60 años, o sea personas aún en edad laboral. En el Instituto de Ciencias Neurológicas "Oscar Trelles Montes" la enfermedad cerebrovascular es la primera causa de 
hospitalización; el costo de hospitalización de los pacientes con enfermedad cerebrovascular es elevado por todos los cuidados especiales, por los exámenes auxiliares especializados, la medicación casi permanente en todos los casos y la estancia prolongada [3] .De todas las enfermedades cerebrovasculares la mortalidad fue del $7 \%$ para las enfermedades isquémicas y del $15 \%$ para las enfermedades hemorrágicas [8] .

El avance en el tratamiento de la IC humana se hace posible gracias al conocimiento de la fisiopatología de la enfermedad que conlleva al descubrimiento de fármacos neuroprotectores eficaces que eviten la progresión del infarto y , a su vez, rescaten la zona del tejido cerebral que, con el trascurso del tiempo podría incorporarse al núcleo del infarto ,zona de penumbra isquémica .La mejor herramienta de que se dispone hoy para el estudio de la fisiopatología de la IC son los modelos experimentales descritos hasta la actualidad, que permiten abordar de manera simple el cuidado de esta afección que se caracteriza por su complejidad y heterogeneidad, el uso y producción de modelos animales en la investigación biomédica permiten esclarecer los mecanismos básicos del mantenimiento, pérdida y restablecimiento de la salud, siendo este el paso previo al desarrollo de nuevas estrategias terapéuticas [9] .

Los animales de experimentación más utilizados son el jerbo y la rata siendo esta última la preferentemente empleada pues el jerbo es extremadamente pequeño y se hace difícil la monitorización de los parámetros fisiológicos y aumenta la tendencia en presentar epilepsia [10].

Existen, experimentalmente dos tipos básicos de isquemia cerebral, global y focal. La isquemia global reproduce la lesión cerebral aparecida tras un paro cardíaco que ha tenido lugar en el ser humano; la isquemia focal surge de la oclusión de arterias específicas que irrigan un área anatómica del cerebro y que aparece en diversos procesos patológicos, como enfermedad cerebrovascular isquémica, hemorragia subaracnoidea y el traumatismo craneoencefálico [11].

En la actualidad, los modelos experimentales más utilizados son los de isquemia cerebral focal en ratas consistentes en la oclusión de la arteria cerebral media tanto a la altura de sus ramas corticales como de su origen por vía intraluminal y los modelos in vitro $[12,13,14]$.

Kelly (2001) y Levine (1966), presentaron técnicas relacionadas a oclusión de arterias carótidas comunes y exsanguinación, datos que hemos utilizado en nuestro trabajo para adaptarlos y verificar por análisis histopatológico los efectos neurológicos que se observan, considerando que cuanto más sencillo es un modelo experimental, más simple es estudiar los procesos fisiopatológicos subyacen patología investigada $[11,12,13,15]$.

Considerando que las investigaciones sobre este campo son ilimitadas, no solo por la trascendencia y costo social de la enfermedad isquémica cerebral, los estudios de nuevas metodologías experimentales permitirán alcanzar mejores herramientas con rigor científico para el conocimiento fisiopatológico, terapéutico y preventivo de la enfermedad isquémica cerebral.

Por lo expuesto nos propusimos determinar si la ligadura de arteria carótida común izquierda y ex sanguinación podría ser utilizada como modelo de isquemia cerebral focal en ratas.

\section{Material y Método}

Se usaron ratas albinas, Rattus rattus var. Norvegirus machos, de 8 -9 semanas de edad, con peso de 180-200 +- 20 gr. Se conformaron 2 grupos, de 16 animales cada grupo, según tratamiento estadístico [20,21], distribuidos de la siguiente manera:

- $\quad$ Grupo Control (Sham): es el grupo en el que se realiza la incisión en el cuello y se sutura sin realizar la ligadura de arteria ni la exsanguínea transfusión.

- Grupo Experimental (operado): es el grupo que se liga la arteria carótida común izquierda y ex sanguínea transfusión.

El Diseño del estudio fue experimental aleatorio simple de dos grupos. Se describe el procedimiento experimental y el equipamiento utilizado. Aquí no se muestran los resultados sino se describe la metodología empleada en el trabajo experimental.

\section{Procedimiento}

Se anestesió a los animales con $5 \mathrm{mg} / \mathrm{kg}$ de hidrocloruro de xilazina y $50 \mathrm{mg} / \mathrm{kg}$ de clohidrato de Ketamina por vía intraperitoneal, y se les dejó reposar hasta que los anestésicos hicieran efecto [22].

Se procedió a la sangría por punción cardiaca, y se extrajo de cada rata el $10 \%$ del volumen de sangre circulante [23]. 
Bajo condiciones de asepsia y antisepsia, se realizó una incisión en la línea media del cuello y diseccionó la piel, tejido subcutáneo y músculo hasta lograr identificar la arteria carótida común (ACC) izquierda y se ligó con sutura Nylon 5-0, para interrumpir la circulación sanguínea de manera ipsilateral, se suturó la herida y se administró lidocaína, finalmente se dejó descansar al animal a una temperatura ligeramente mayor a la ambiental en cámaras previamente confeccionadas [10,23,24,25].

Se colocaron termistores colónicos para monitorización de la temperatura corporal, durante 24 horas.

\subsection{Obtención y preparación del material de estudio}

Los animales después de 24 horas de realizada la cirugía fueron sacrificados, por sobredosis de anestésico (Ketamina).

Se procedió a la decapitación en un nivel justo por encima de las escápulas. Una vez que la cabeza fue removida se expuso el interior del cráneo separando los huesos nasales y frontales en sentido sagital, para luego a extraer los huesos de la bóveda craneana. Después se extrajo con cuidado el encéfalo y se sumergió en formol al $10 \%$ para su conservación [24,26].

Los cerebros se fijaron en solución de formol y fueron embebidos en parafina para posteriormente ser sometidos a la tinción con hematoxilina-eosina.

A fin de poder evaluar comparativamente todas las muestras se realizaron secciones coronales de cerebro en tres regiones (Neocortex, Hipocampo, ganglios basales) por ser las áreas más vulnerables a daño en una lesión isquémica.

En la región del hipocampo se analizó las características de las neuronas oscuras (contracción celular prominente, basofilia, dendritas apicales en sacacorchos), mientras que en el Neocortex y los ganglios basales se analizó las características de las neuronas rojas (citoplasma brillante, rojo o rosado, núcleo picnótico desintegrado, dendritas apicales en sacacorchos, nucléolo poco notorio, gránulos de nissl poco notorios, contracción celular prominente).

Para determinar el daño se realizó un conteo de las características alteradas en cada una de las áreas del cerebro de cada animal del grupo de estudio y se aplicó una prueba estadística que permitió establecer diferencias significativas entre grupos [4].

\subsection{Análisis estadístico}

Los datos fueron procesados con los paquetes estadísticos SPSS 15.0 y Minitab Statistical Software; previa elaboración de la base de datos correspondiente. Para las variables cuantitativas se calculó el promedio y su correspondiente varianza, realizándose gráficos de barras simples para resaltar los hallazgos de interés.

Se utilizó la prueba de Kolmogorov-Smirnov para verificar la normalidad de una distribución y luego se determinó si existe diferencia significativa entre los promedios de los grupos, para esto se aplicó el Análisis de Varianza (ANOVA); si existe diferencias significativas ( $p$-valor $<0.01$ ), quiere decir que al menos dos comparaciones son diferentes.

\section{Resultados y discusión}

La Fig. 1 representa las características neuronales que indican daño celular en tres zonas del cerebro (Neocortex, hipocampo y ganglio basal) en cada uno de los grupos.

El análisis estadístico muestra que el grupo experimental (ICF), presentó una media de 6.4 con un error típico de 0.68 , mientras que el grupo control (Sham) obtuvo una media de 3.2 con un error típico de 0.55 .

En la comparación entre grupos, se encontró diferencias altamente significativas entre los grupos control (Sham) y experimental (ICF) con un p 0.001 .

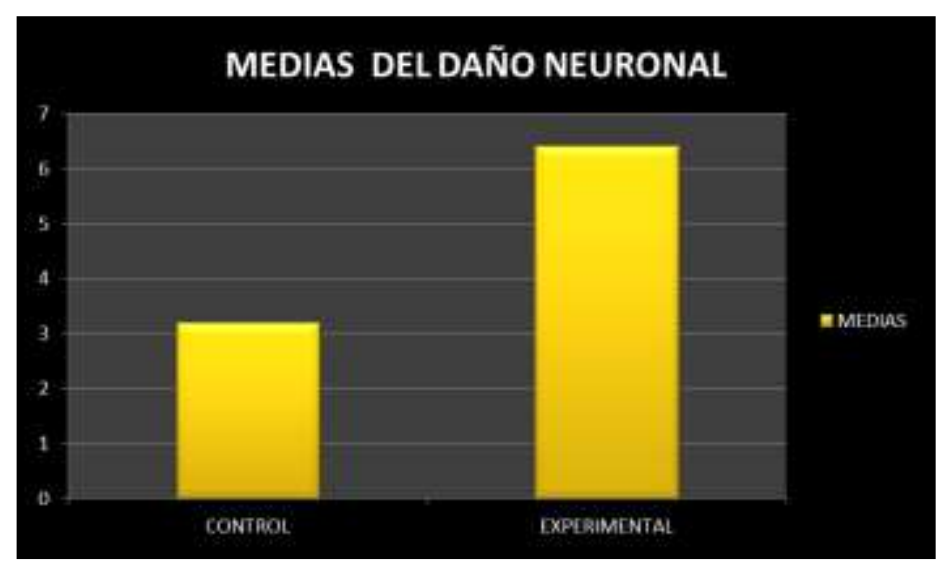

Figura 1: Representación gráfica de la Medias del daño Neurológico evidenciado por media del número de las disconformidades en los Grupos Control- SHAM y Experimental-ICF, pr 0.001

Media (Control) $\quad 3.2$ Media (Experimental) $\quad 6.4$ 
Control N: $1.5 \quad \mathrm{H}: 1.5 \quad$ GB: 0.2

Experimental $\quad \mathrm{N}: 0.2 \quad \mathrm{H}: 0.2 \quad \mathrm{~GB}: 1.5$

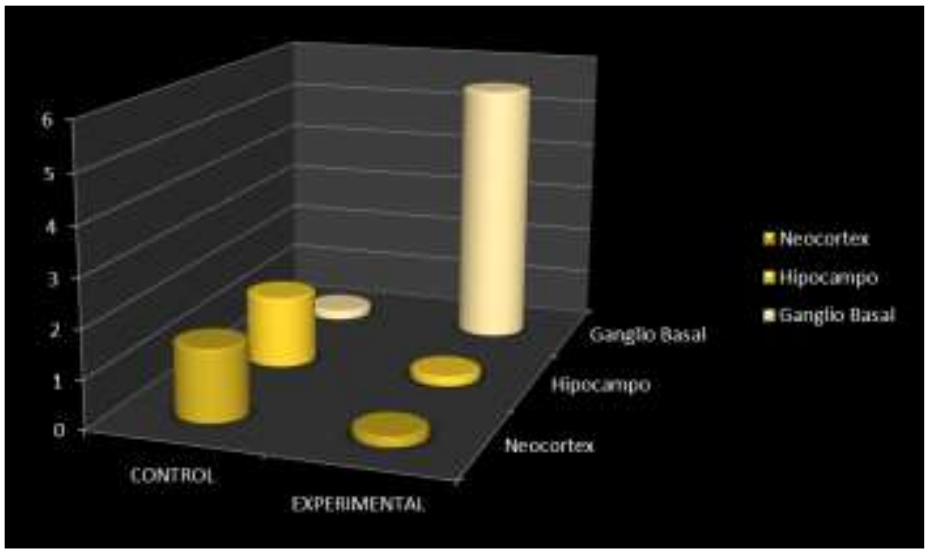

Figura 2: $\quad$ Representación gráfica de la Medias del daño Neurológico evidenciado por media del número de las disconformidades en los Grupos Control- SHAM y Experimental-ICF, según la zona cerebral afectada, con $p<0.01$.

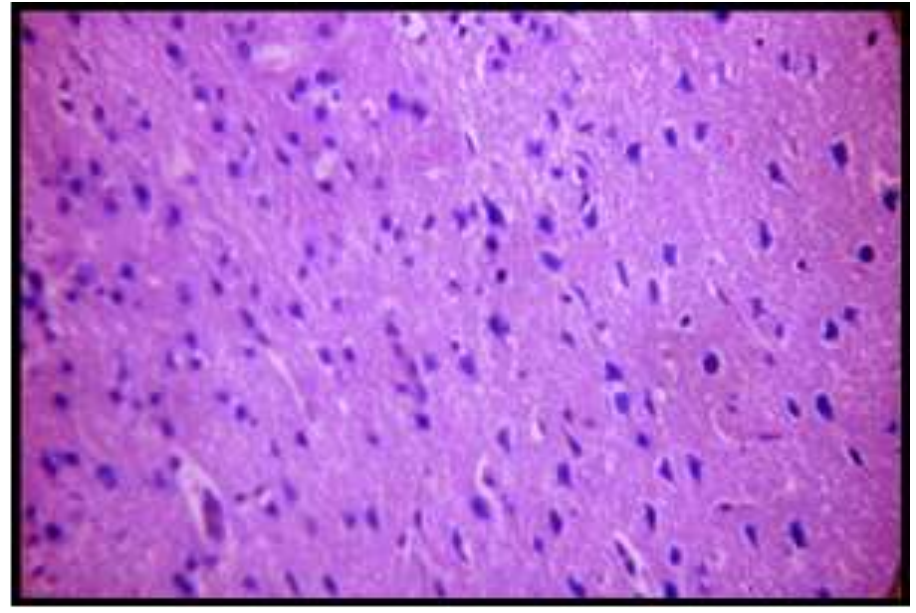

Fig. 3: Neuronas oscuras del hipocampo - Grupo Control

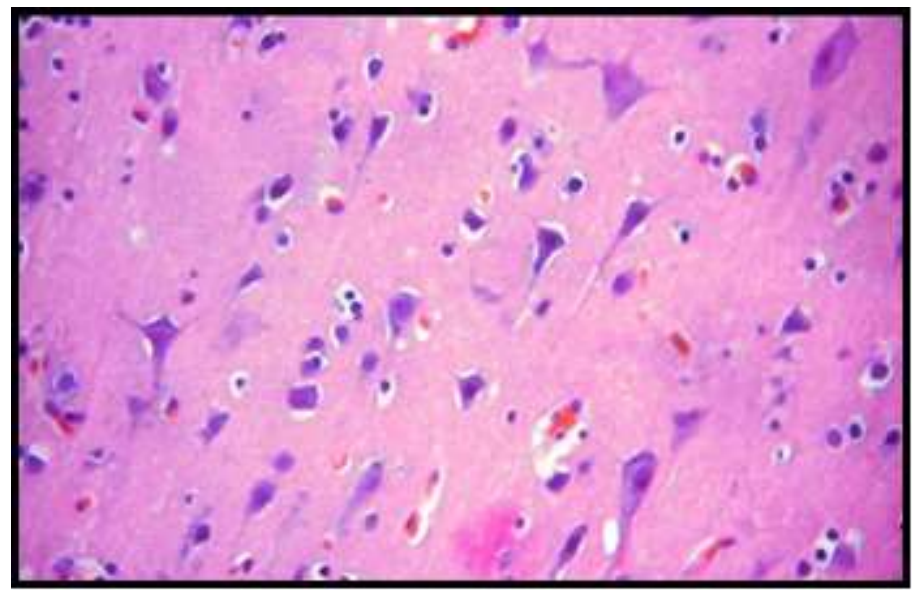

Fig. 4: Contracción celular prominente en neuronas rojas de Neocortex cerebral - Grupo Experimental
En la Fig. 1 se representa las características neuronales que indican daño celular en tres zonas del cerebro más sensibles a la disminución de oxígeno (Neocortex, hipocampo y ganglio basal) en el grupo Control y Experimental, mostrando que en el grupo Experimental se encuentra el mayor valor con una media de 6.4 con datos estadísticas significativas, $p<0.01$.

Estos datos evidencian que la ligadura de arteria carótida común y exsanguinación presenta daños neurológicos localizados o focales. Estos datos coinciden con las investigaciones realizadas por Quinzaños. Relacionamos estos hallazgos a la oclusión de las arterias asociada a la hipoxia generada por la disminución del flujo sanguíneo cerebral, y que produce daño neuronal ipsilateral a la oclusión [24, 29, 30,31].

La valoración histológica de la lesión isquémica es la más comúnmente empleada, en nuestros datos encontramos que el área más afectada fueron los ganglios basales por presentar el mayor número de disconformidades como son basofilia en neuronas oscuras y citoplasma brillante, dendritas apicales en sacacorchos, nucléolo poco notorio y contracción celular prominente en las neuronas rojas( Fig. 3 ) lo que se aleja de los hallazgos de Brint (1988), que encontró que la zona más afectada fue la capa CA1 del hipocampo y Tamura ( 1981) reporta alta incidencia de daños en el neocórtex y núcleo estriado $[24,32,33,34,35,36]$.

\section{Conclusiones}

- La ligadura de arteria carótida común izquierda y ex sanguinación produce daños neuronales localizados.

- La zona más afectada son los ganglios basales con $p<0.01$.

\section{Referencias}

[1] P.Wolf et al. Secular trends in stroke incidence and mortality. The Framingham Study. Stroke. 23(2000)1551-5

[2] C. Arango-Dávila et al. Fisiopatología de la isquemia cerebral focal: aspectos básicos y proyección a la clínica. Rev Neurol 39(2004):156-65

[3] A.Garate-Salazar et al. Enfermedad isquémica .Propuesta de protocolo de manejo. Revista Peruana de Neurología.8 (2002) 16-23.

[4] J.Quinzaños et al. Efectos neurológicos de la ligadura de la carótida común izquierda e hipoxia inducida en ratas neonatas. Cir Ciruj; 
176 (2008) 119-125

[5] R. Prieto-Arribas et al. Modelos experimentales de isquemia cerebral. Rev Neurol 47 ( 2008) 414-26

[6] OMS. Factores de riesgo de enfermedad cardiovascular: Nuevas esferas de investigación.2010

[7] J.Garvi et al. Modelos experimentales de isquemia cerebral. Rev neurol; 47(2008) 41426.

[8] Oficina de Estadistica e Informática del Instituto de Ciencias Neurológicas Oscar Trelles Montes. 2000

[9] J.Roda et al. Modelos experimentales en isquemia cerebral. Neurologia.13(1998) 9

[10] S.Levin et al. Effects of ischemia and other procedures on the brain and retina of the gerbil. Exp, 16(1996) 255-262

[11] A.Tamura et al. Focal cerebral isquemia in the rat.J Cereb Blood Flow Metab ; 1(1981) 53-60

[12] TH.Liu. Polyethylene glycol-conjugated superoxide dismutase and catalase reduce ischemic brain injury. Am $\mathrm{J}$ Physiol 256(1989): H589-93.

[13] J. Kouzumi. Experimental studies of ischemic brain edema. A new experimental model of cerebral embolism in rats in which recirculation can be introduced in the ischemic area. Jpn J Stroke ,8(1986) 1-8.

[14] MJ. Jano et al. Guía para el Cuidado y Uso de los Animales de Laboratorio. Institute of Laboratory Animal Resources.1999; México

[15] B. Rollin. The Experimental Animal in Biomedical Research: Care, Husbandry, and Wellbeing, An Overview by Species. eds. Boca Raton, Fla.: CRC Press.2 (1995).

[16] A.Quezada. Introducción al manejo de animales de laboratorio: roedores y pequeñas especies. Ediciones de la Universidad Autónoma de Yucatán, Unidad de Ciencias Biomédicas México.1997.

American Physiological Society.Guiding principles for research involving animals and humans beings. Am J Physiol Regul Integr

[17] Comp Physiol 283(2002): R281-R283 Institute For Laboratory Animal Research (ILAR). Guide for the Care and Use of Laboratory Animals. National Academy Press,

[18] Washington, DC.1996.

D.Montgomery. Diseño y Análisis de Experimentos.2da edición. Editorial Limusa. 2002; 647

[19] R.Steel et al. Principios y Procedimientos de Bioestadistica.Mc Graw-Hill. Mexico.1996; 2da Edición.

[20] F.Viniegra et al.. Anestesia en Ratas. AMMVEPE.13 (2002):27-31.

$\mathrm{K}$. Sugio et al. A new model of bilateral

[21] hemispheric ischemia-modified four-vessel occlusion in rats. Stroke .1998; 19: 922

[22] M.López-Hernandez et al. Cerebral ischemia: some secondary alterations and animal models. Arch Neurocien (Mex) .10(2005)3:

[23] 160-167

WA. Pulsinelli et al. A new model of bilateral hemispheric ischemia in the anaesthetized rat. Stroke.10 (1979) 267-72.

[24] E. Arch et al. Efectos de la ligadura de la arteria carótida común en los valores gasométricos en cobayos. An Orl Mex.

[25] 53(2008)6-12.

E-mail: luzguerrero-61@hotmail.com 